\title{
Resource Allocation for AF Relaying Wireless-Powered Networks with Nonlinear Energy Harvester
}

\author{
Tien-Tung Nguyen, Van-Dinh Nguyen, Quoc-Viet Pham, Jong-Ho Lee, Yong-Hwa Kim
}

\begin{abstract}
This letter considers a relay-based wireless-powered communication network to assist wireless communication between a source and multiple users. In particular, the relay adopts a nonlinear energy model to harvest energy from a power beacon and subsequently uses it for information transmission over timedivision multiple access. Aiming at the maximization of end-toend (e2e) sum throughput, we formulate a novel optimization problem that jointly optimizes the power and time fraction for energy and information transmission. For a simple yet efficient solution for the nonconvex problem, we first convert it to a more computationally tractable problem and then develop an iterative algorithm, in which closed-form solutions are obtained at each iteration. The effectiveness of our proposed approach is verified and demonstrated through simulation results. Moreover, the results reveal that the source should transmit with its maximum allowable power budget to obtain the optimal e2e sum throughput.
\end{abstract}

Index Terms-Amplify-and-forward relay, nonlinear energy harvester, resource allocation, wireless-powered communications.

\section{INTRODUCTION}

$\mathbf{R}$ ECENTLY, simultaneous wireless information and power transmission (SWIPT) exploiting the characteristics of radio frequency (RF) signals, which can carry both energy and information, has been advocated as a promising technique to extend the lifetime of wireless networks [1], [2]. However, the RF energy harvested at the end devices is often limited due to the significant attenuation of electromagnetic wave propagation in wireless channels. Consequently, SWIPT systems are practically suited for short-range communications. As an alternative, the power beacon (PB)-based technique has been proposed to support wireless power transfer (WPT) systems [3]-[5]. Low-cost PBs can be deployed much closer to wireless-powered devices to improve the system efficiency.

This work was supported by the National Research Foundation of Korea (NRF) funded by Korea government (MSIT) (2019R1A2C2086621). (Corresponding author: Yong-Hwa Kim).

The work of V.-D. Nguyen was supported in part by the Luxembourg National Research Fund (FNR) in the framework of the FNR-FNRS bilatera project "InWIP-NETs: Integrated Wireless Information and Power Networks."

T.-T. Nguyen is with the Department of Electronic Engineering, Myongji University, Yongin 17058, Korea and Telecommunication Division, Industria University of Ho Chi Minh City, Ho Chi Minh City 700000, Vietnam (e-mail: nguyentientung@iuh.edu.vn).

V.-D. Nguyen is with the Interdisciplinary Centre for Security, Reliability and Trust (SnT), University of Luxembourg, L-1855 Luxembourg City, Luxembourg (e-mail: dinh.nguyen@uni.lu).

Q.-V. Pham is with the Research Institute of Computer, Information and Communication, Pusan National University, Busan 46241, Korea (e-mail: vietpq@pusan.ac.kr).

J.-H. Lee is with the School of Electronic Engineering, Soongsil University, Seoul 06978, Korea (e-mail: jongho.lee@ssu.ac.kr).

Y.-H. Kim is with the Department of Electronic Engineering, Myongji University, Yongin 17058, Korea. (e-mail: yongkim@mju.ac.kr).
Moreover, relay-based wireless-powered communication networks (RWPCNs) have attracted significant research interest, as they help extend both the lifetime and communication coverage of wireless networks [6]-[9]. In [6], the sum throughput maximization problem of multi-user wireless-powered communication networks (WPCNs) adopting amplify-andforward (AF) and decode-and-forward (DF) relays was investigated, where the energy harvesting $(\mathrm{EH})$ duration in the downlink (DL) wireless energy transfer (WET) and the energy expenditure at the relay for each time slot in the uplink (UL) wireless information transfer (WIT) were jointly optimized. Similar to [6], the authors in [7] studied a relay-assisted multi-user system, in which the DF relay not only provides energy for users in the DL WET, but also helps users transmit information to the base station (BS) in the UL WIT. Aiming at maximizing the sum throughput for multiple DF relays in a WPCN, an effective algorithm for optimizing the time allocation and EH power coefficients was developed in [8]. Very recently, the work in [9] proposed an iterative algorithm to obtain a suboptimal solution for the optimization problem of max-min end-to-end (e2e) rate for a multi-pair SWIPT DF relaying network. However, the works cited above (i.e., [6][8]) mainly focused on UL WIT, and ignored the benefit of power allocation (PA) at the relay. In addition, the iterative algorithm developed in [9] was heavily based on a large number of approximate functions, and the convex program at each iteration was solved using existing solvers, making it unimplementable in practice. Thus, we investigate a joint design of power and time fraction for EH so that the system performance is remarkably enhanced.

In this study, we consider an RWPCN system, in which a BS communicates with multiple users through an AF relay wirelessly powered by a PB. Our objective is to maximize the e2e sum throughput of the system by jointly optimizing the power and time fraction for energy and information transmission. In contrast to [6]-[8], we study the PA at the relay for the DL WIT to serve multiple users under a practical EH model. Through time-division multiple access (TDMA) and the AF protocol, the PA in our problem becomes more complex than in [9] due to the strong coupling between the optimization variables and the presence of a fractional form in the e2e throughput function. Owing to the nonconvexity of the formulated problem, we first transform it into a more computationally tractable form and then propose a very efficient iterative algorithm for its solution. Unlike [9], the proposed algorithm does not rely on any existing convex solvers owing to closed-form solutions obtained in each iteration. Simulation results are provided to demonstrate the effectiveness of the proposed algorithm in terms of e2e sum throughput. 


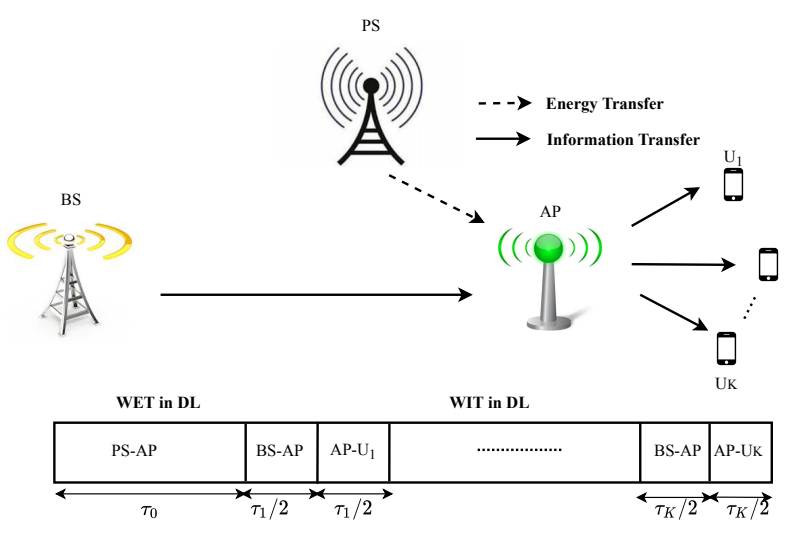

Fig. 1. Illustration of an RWPCN

\section{System Model AND PROBlem DESCRIPTION}

\section{A. System Model}

We consider a two-hop relaying network consisting of one source (i.e., BS) and $K$ users, as illustrated in Fig. 1. The set of users is denoted by $\mathcal{K}=\{1, \cdots, K\}$ and the $k$ th user is denoted by $\mathrm{U}_{k}$. In this work, we assume that the BS and users are geographically separated, i.e., there is no direct communication link between them. In this study, the information is transmitted via an energy-constrained $\mathrm{AF}$ relay, which is referred to as an access point (AP). The BS is equipped with $N_{T}$ antennas, whereas the other nodes have a single antenna. The energy-constrained AF relay is powered by a single-antenna power station (PS). The channels of the PS-AP, BS-AP, and AP-U $\mathrm{U}_{k}$ links are denoted as by $h_{P} \in \mathbb{C}, \mathbf{h} \in \mathbb{C}^{1 \times N_{T}}$, and $g_{k} \in \mathbb{C}$, respectively. We further assume that perfect channel state information (CSI) of all the links is available at the transmitters [6]-[9]. All the channels experience quasi-static flat fading and are assumed to be constant during each transmission time-block.

The transmission time-block structure for a TDMA-based RWPCN is depicted in Fig. 1. Without loss of generality, the transmission time-block is normalized to be a unit. The entire process is divided into two consecutive phases, called the DL WET and DL WIT phases. In the WET phase, the PS powers the AP, whereas in the WIT phase, the information transmission from the BS to the users occurs in two hops.

\section{B. WET Phase}

We adopt the "harvest-then-transmit" protocol at the relay for the EH in the WET phase with the time duration $\tau_{0}$ [6], [7]. Considering the practical EH model [10], [11], the energy harvested at the AP can be calculated as $E_{\text {nln }}=\Theta_{0} \tau_{0}$, where $\Theta_{0} \triangleq \frac{\pi-M v}{1-v}, v \triangleq \frac{1}{1+\exp (a b)}$, and $\pi \triangleq \frac{M}{1+\exp \left(-a\left(P_{i n}-b\right)\right)}$. Here, $P_{i n} \triangleq P_{E}\left|h_{P}\right|^{2}$ is the total input power at the AP, and $P_{E}$ is the transmit power of the PS. $M, a$, and $b$ denote the maximum amount of harvested power when the EH circuit reaches saturation, the nonlinear charging rate related to the input power, and the nonlinearity characteristic of the diode, respectively.

\section{WIT Phase}

For $\mathrm{U}_{k}$ in the first half of the subtime-slot $\tau_{k}$, the BS transmits a signal $x_{k}$, with $\left|x_{k}\right|^{2}=1$, to the AP with transmit power $P_{I}$ using maximum ratio transmission (MRT). Thus, the received signal at the AP is expressed as

$$
r_{\mathrm{AP}_{k}}=\mathbf{h}^{H} \mathbf{w} x_{k}+n_{a},
$$

where $\mathbf{w} \triangleq \sqrt{P_{I}} \frac{\mathbf{h}}{\|\mathbf{h}\|} \in \mathbb{C}^{N_{T} \times 1}$ is the MRT beamforming vector at the $\mathrm{BS}$ for transmitting the signal $x_{k}$, and $n_{a}$ is the additive white Gaussian noise (AWGN) at the AP with zero-mean and variance $\delta_{a}^{2}$. Next, the AP adopts the AF protocol to convey the amplified version of the signal with an amplification coefficient $\Omega \triangleq \sqrt{\frac{1}{P_{I}\|\mathbf{h}\|^{2}+\delta_{a}^{2}}}$ to $\mathrm{U}_{k}$. Thus, the signal received at $\mathrm{U}_{k}$ is expressed as

$$
r_{k}=\sqrt{p_{k}} g_{k}\left(\Omega r_{\mathrm{AP}_{k}}\right)+n_{k},
$$

where $n_{k} \sim \mathcal{C N}\left(0, \delta_{k}^{2}\right)$ and $p_{k}$ is the transmission power of the AP allocated to $\mathrm{U}_{k}$.

Thus, the achievable e2e throughput of $\mathrm{U}_{k}$ is given as

$$
R_{k}^{T}=W \frac{\tau_{k}}{2} \log \left(1+\omega_{k}^{T}\right)
$$

where $W$ is the system bandwidth and $\omega_{k}^{T} \triangleq$ $\frac{P_{I} p_{k}\|\mathbf{h}\|^{2}\left|g_{k}\right|^{2}}{p_{k}\left|g_{k}\right|^{2} \delta_{a}^{2}+P_{I}\|\mathbf{h}\|^{2} \delta_{k}^{2}+\delta_{a}^{2} \delta_{k}^{2}}$.

\section{Problem Formulation}

The maximization problem of the e2e sum throughput of the AF-based RWPCN can be mathematically formulated as follows:

$$
\begin{aligned}
& \text { (T-P1) : } \max _{\tau_{0},\left\{\tau_{k}\right\},\left\{p_{k}\right\}, P_{I}} \sum_{k=1}^{K} W \frac{\tau_{k}}{2} \log \left(1+\omega_{k}^{T}\right) \\
& \text { s.t. C1 : } \sum_{k=0}^{K} \tau_{k} \leq 1, \tau_{k} \in(0,1), \forall k \text {, } \\
& \text { C2 }: \sum_{k=1}^{K} \frac{\tau_{k}}{2} p_{k} \leq \Theta_{0} \tau_{0}, \forall k, \text { C3 }: p_{k} \geq 0, \forall k, \\
& \text { C4 : } P_{I} \leq P_{\mathrm{BS}} \text {, }
\end{aligned}
$$

where $P_{\mathrm{BS}}$ is the maximum allowable power budget at the BS. Constraint $\mathrm{C} 2$ indicates that the total energy consumption of the AP in the WIT phase should not be larger than the harvested energy in the WET phase. Thus, the forms of the objective and C2 make the problem (4) different from that in [6]-[9].

Lemma 1: $R_{k}^{T}$ is a concave function of two variables $p_{k}$ and $\tau_{k}$.

Proof: By checking the sign of the second-order derivative of $R_{k}^{T}$ corresponding to each variable, it can be verified that $R_{k}^{T}$ is a concave function. However, in $\mathrm{C} 2$, there exists a strong coupling of the optimization variables, making (T-P1) a nonconvex optimization problem.

\section{PROpOSED SOLUTION}

In this section, we provide an efficient solution for the maximization problem of e2e sum throughput in (4) of the AF-assisted RWPCN. To do, we first determine the optimal transmit power $P_{I}^{*}$ of the BS. After verifying the sign of the first-order derivative of the objective function in (T-P1) for 
$P_{I}$, we observe that the objective function is an increasing function of $P_{I}$. Thus, the e2e sum throughput only attains the maximum value at the point $P_{I}^{*}=P_{\mathrm{BS}}$.

Subsequently, for a given $P_{I}^{*}$, we transform the optimization problem to a convex problem. Let us denote $A \triangleq P_{\mathrm{BS}}\|\mathbf{h}\|^{2}$, $B \triangleq \delta_{a}^{2}, C \triangleq\left(P_{\mathrm{BS}}\|\mathbf{h}\|^{2}+\delta_{a}^{2}\right) \delta_{k}^{2}$, and $\gamma_{k} \triangleq\left|g_{k}\right|^{2}$. By introducing an auxiliary variable $e_{k} \triangleq p_{k} \tau_{k}$, (T-P1) can be rewritten equivalently as

$$
\begin{array}{r}
(\mathrm{T}-\mathrm{P} 2): \max _{\tau_{0},\left\{\tau_{k}\right\},\left\{e_{k}\right\}} W \sum_{k=1}^{K} \frac{\tau_{k}}{2} \log \left(1+\frac{A \frac{e_{k} \gamma_{k}}{\tau_{k}}}{B \frac{e_{k} \gamma_{k}}{\tau_{k}}+C}\right) \\
\text { s.t. C1, } \overline{\mathrm{C}} 2: \sum_{k=1}^{K} e_{k} \leq 2 \Theta_{0} \tau_{0}, \forall k, \overline{\mathrm{C}} 3: e_{k} \geq 0, \forall k .
\end{array}
$$

We introduce the following proposition to evaluate (T-P2), whose proof is given in Appendix A.

Proposition 1: (T-P2) is a convex optimization problem.

Towards an efficient solution, we first determine the optimal information transmission time allocation (ITA) $\tau_{k}^{*}, \forall k \in \mathcal{K}$ with the given $e_{k}$ and $\tau_{0}$, and then derive the closed-form solution for the optimal energy allocation (EA) $e_{k}^{*}, \forall k \in \mathcal{K}$ with the given $\tau_{0}$ and $\tau_{k}^{*}$. Finally, based on a golden search method, we determine the optimal solution $\tau_{0}^{*}$. First, with the given $\tau_{0}$ and $e_{k}$, we consider (T-P2) with respect to $\tau_{k}$. By Proposition 1, the Lagrangian of (T-P2) is given as

$$
L(\boldsymbol{\tau}, \mu)=\sum_{k=1}^{K} R_{k}^{T}-\mu\left(\sum_{k=0}^{K} \tau_{k}-1\right),
$$

where $\tau \triangleq\left\{\tau_{k}\right\}$, and $\mu$ is the non-negative Lagrangian multiplier associated with constraint $\mathrm{C} 1$. Moreover, to obtain the optimal solution, the Karush-Kuhn-Tucker conditions should be satisfied as follows: $\mu^{*}\left(\sum_{k=0}^{K} \tau_{k}^{*}-1\right)=0$,

$$
\begin{aligned}
\frac{\partial L(\boldsymbol{\tau}, \mu)}{\partial \tau_{k}}= & \frac{W}{2} \log \left(1+\frac{A e_{k} \gamma_{k}}{B e_{k} \gamma_{k}+\tau_{k} C}\right) \\
& -\frac{W \tau_{k} A C e_{k} \gamma_{k}}{2\left(B e_{k} \gamma_{k}+\tau_{k} C\right)\left(A e_{k} \gamma_{k}+B e_{k} \gamma_{k}+\tau_{k} C\right)}
\end{aligned}
$$

$$
-\mu^{*}=0 \text {. }
$$

The condition $\left(\sum_{k=0}^{K} \tau_{k}^{*}-1\right)=0$ always holds for the optimal solution, and it results in $\mu^{*}>0$. Let us denote $u \triangleq \frac{A e_{k} \gamma_{k}}{B e_{k} \gamma_{k}+\tau_{k} C}$. Then, (7) is calculated as

$$
\frac{\partial L(\boldsymbol{\tau}, \mu)}{\partial \tau_{k}}=\log (1+u)-\frac{u(A-B u)}{A(u+1)}-\frac{2 \mu^{*}}{W}=0 .
$$

It can be verified that $y(u)=\log (1+u)-\frac{u(A-B u)}{A(u+1)}$ is a monotonically increasing function of $u$ due to $y^{\prime}(u)=$ $\frac{2 B u^{2}+(A+B) u}{A(u+1)^{2}}>0$. Consequently, a unique solution exists for $y(u)-\frac{2 \mu^{*}}{W}=0$. Thus, $\frac{A e_{1} \gamma_{1}}{B e_{1} \gamma_{1}+\tau_{1} C}=\ldots=\frac{A e_{K} \gamma_{K}}{B e_{K} \gamma_{K}+\tau_{K} C}$, i.e.,

$$
e_{1} \gamma_{1} / \tau_{1}^{*}=\ldots=e_{K} \gamma_{K} / \tau_{K}^{*}
$$

Given $\tau_{0}$, from (9) and $\sum_{k=0}^{K} \tau_{k}^{*}-1=0$, the optimal ITA $\tau_{k}^{*}$ is

$$
\tau_{k}^{*}=\left(1-\tau_{0}\right) e_{k} \gamma_{k} / \sum_{k=1}^{K} e_{k} \gamma_{k}, \forall k .
$$

Subsequently, by substituting $\tau_{k}^{*}$ into (5), a simpler form of (T-P2) is

$$
\text { (T-P3) : } \Upsilon\left(\tau_{0}, \mathbf{e}\right)\left[\text { s.t. } \overline{\mathrm{C}} 2: \sum_{k=1}^{K} e_{k} \leq 2 \Theta_{0} \tau_{0}, \overline{\mathrm{C}} 3\right] \text {, }
$$

where $\mathbf{e} \triangleq\left\{e_{k}\right\}$ and $\Upsilon\left(\tau_{0}, \mathbf{e}\right) \triangleq \max _{\tau_{0}, \mathbf{e}} W\left(\frac{1-\tau_{0}}{2}\right) \log (1+X)$ with $X \triangleq \frac{A \sum_{k=1}^{K} e_{k} \gamma_{k}}{B \sum_{k=1}^{K} e_{k} \gamma_{k}+\left(1-\tau_{0}\right) C}$.

Given $\tau_{0}$, (T-P3) is considered a nonconcave problem with respect to $e_{k}$ by checking the sign of the second-order derivative of its objective. We provide the following lemma to determine the optimal EA at the AP, where the proof is detailed in Appendix B.

Lemma 1: Given $\tau_{0}$, the optimal EA is obtained as

$$
e_{k, 1}^{*}=\left[\frac{\left(1-\tau_{0}\right) \gamma_{k}\left(\sqrt{\Lambda_{k}}+(A+2 B) C\right)+V_{-k}}{2}\right]^{+},
$$

or

$$
e_{k, 2}^{*}=\left[\frac{\left(1-\tau_{0}\right) \gamma_{k}\left(\sqrt{\Lambda_{k}}-(A+2 B) C\right)-V_{-k}}{2}\right]^{+},
$$

where $\Lambda_{k} \triangleq(A C)^{2}+\frac{2 W A B(B+A) C \gamma_{k}}{\vartheta}, \quad V_{-k} \triangleq$ $\left(2 B(B+A) \sum_{i=1, i \neq k}^{K} e_{i} \gamma_{i}\right) \gamma_{k}$, and $[y]^{+} \triangleq \max (y, 0)$. The energy allocation in (12) or (13) is optimal for a given $\vartheta$. The optimal solution $\vartheta$ for the total energy constraint $\overline{\mathrm{C}} 2$ in (T-P2) is determined by a bisection search between $\vartheta_{l}=0$ and a sufficiently large $\vartheta_{h}$. In each iteration, the optimal solution $\mathbf{e}^{*}$ is calculated based on (12) or (13) corresponding to the updated value $\vartheta$ and $\sum_{k=1}^{K} e_{k}^{*} \leq 2 \Theta_{0} \tau_{0}$ for a given $\tau_{0}$.

Subsequently, substituting $e_{k}^{*}$ into (T-P3), we obtain the objective for the variable $\tau_{0}$. As a result, we can use the one-dimensional search method to obtain the optimal duration EH $\tau_{0}$ by calculating $\tau_{0}^{*}=\arg \max \Upsilon\left(\tau_{0}, \mathbf{e}^{*}\right)$. The overall $\tau_{0} \in(0,1)$

process of finding the optimal solution of (T-P1) is summarized in Algorithm 1.

\section{NUMERICAL RESULTS}

In this section, simulation results are provided to demonstrate the effectiveness of our proposed algorithm. Here, the system bandwidth is set to be $1 \mathrm{MHz}$, and the noise power at $\mathrm{AP}$ and $\mathrm{U}_{k}$ is $\delta_{a}^{2}=\delta_{k}^{2}=-100 \mathrm{dBm} / \mathrm{Hz}$. We set $M=24 \mathrm{~mW}$, $a=1500$, and $b=0.0014$ [11]. It is assumed that all the channels are modeled by an exponential Rayleigh distribution with the pathloss exponent of 2.5. The BS, AP, and PS are located at $[0 \mathrm{~m}, 0 \mathrm{~m}],[50 \mathrm{~m}, 0 \mathrm{~m}]$, and $[50 \mathrm{~m}, 10 \mathrm{~m}]$, respectively. The users are randomly placed, assuming that their $\mathrm{x}$ and $y$-coordinate positions are in the range of $[60 \mathrm{~m}, 100 \mathrm{~m}]$ and $[-10 \mathrm{~m}, 10 \mathrm{~m}]$, respectively. 


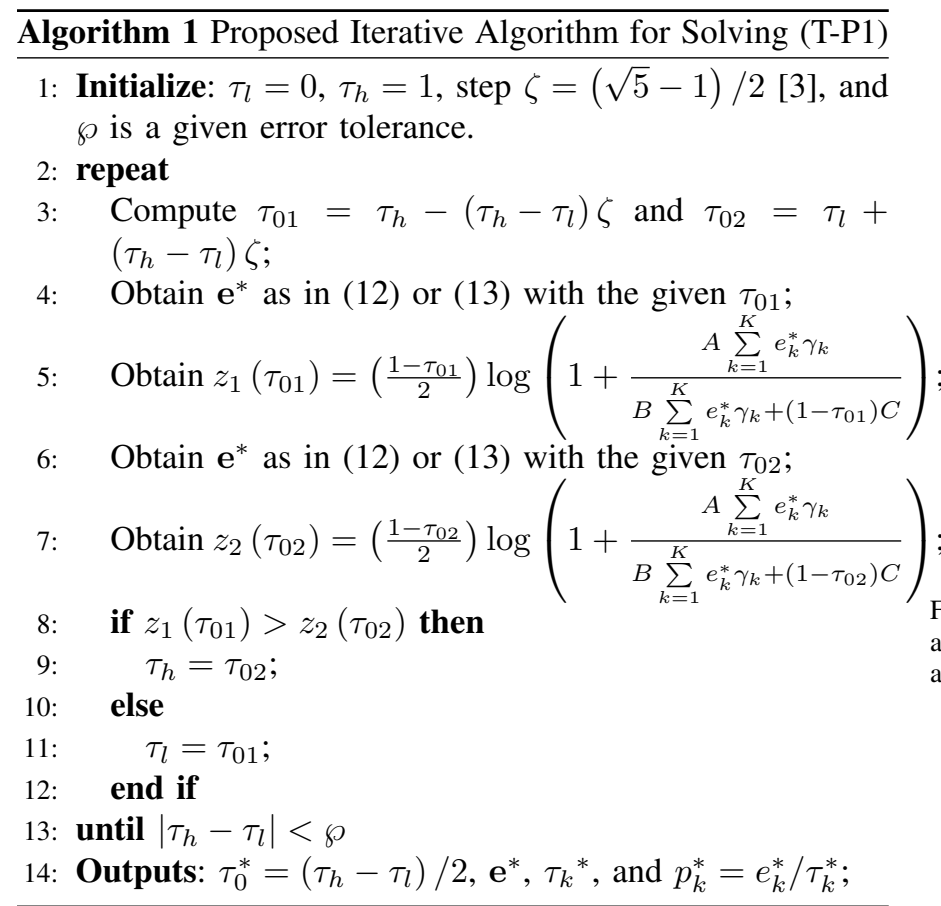

TABLE I

COMPLEXITY ANALYSES OF DIFFERENT SCHEMES

\begin{tabular}{|c|c|}
\hline Scheme & Complexity \\
\hline Proposed scheme & $\mathcal{O}\left(\left(\log _{2}\left(\varsigma^{-1}\right)\right)\left(\log _{2}\left(\wp^{-1}\right)\right)\right)$ \\
\hline EIT scheme & $\mathcal{O}\left(\left(\log _{2}\left(\varsigma^{-1}\right)\right)\left(\log _{2}\left(\wp^{-1}\right)\right)\right)$ \\
\hline FET scheme & $\mathcal{O}\left(\log _{2}\left(\varsigma^{-1}\right)\right)$ \\
\hline
\end{tabular}

In Table I, the complexity analysis of the proposed scheme, equal information time (EIT) allocation and fixed energy time (FET), is presented. For the EIT scheme, the duration of the WIT phase is divided into equal intervals (i.e., $\tau_{1}=\tau_{2}=$ $\left.\ldots=\tau_{K}=\left(1-\tau_{0}\right) / K\right)$ [12]. For the FET scheme, the EH duration is fixed as $\tau_{0}=0.1, \tau_{0}=0.5, \tau_{0}=0.9$ [3]. The two parameters $\varsigma$, and $\wp$ represent the search accuracies of the bisection method for searching the optimal $e_{k}^{*}$ and the golden search method for finding the optimal $\tau_{0}^{*}$, respectively.

Fig. 2 shows the convergence of our proposed algorithm with different numbers of antennas at the BS and different power levels at the PS with $K=10$ and $P_{\mathrm{BS}}=30 \mathrm{dBm}$. Once can see that the e2e throughput is monotonically increased and the algorithm converges after approximately 10 iterations. As expected, the higher the numbers of antennas at the BS and the transmit power at the PS, the better the e2e throughput can be obtained.

In Fig. 3, the e2e sum throughput of our proposed scheme and benchmarking ones change with different transmit powers of the BS is plotted. We can observe that the e2e sum throughput of all the schemes is significantly increased, as the maximum transmit power of the BS increases. However, the performances of two baseline schemes are lower than that of the proposed scheme due to the lack of ITA for the EIT scheme and optimizing $\tau_{0}$ for the FET scheme. This observation confirms the effectiveness of the proposed solution in jointly optimizing the EH duration and the ITA and PA at the AP. In addition, the performance gap between the FET scheme at $\tau_{0}=0.1$ and our proposed scheme is small, as

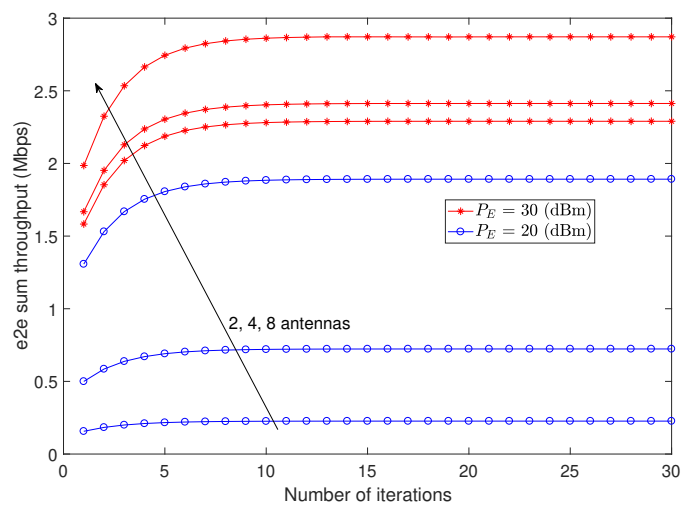

Fig. 2. Convergence of the proposed algorithm with different numbers of antennas at the BS and different power levels at the PS, with $P_{\mathrm{BS}}=30 \mathrm{dBm}$ and $K=10$.

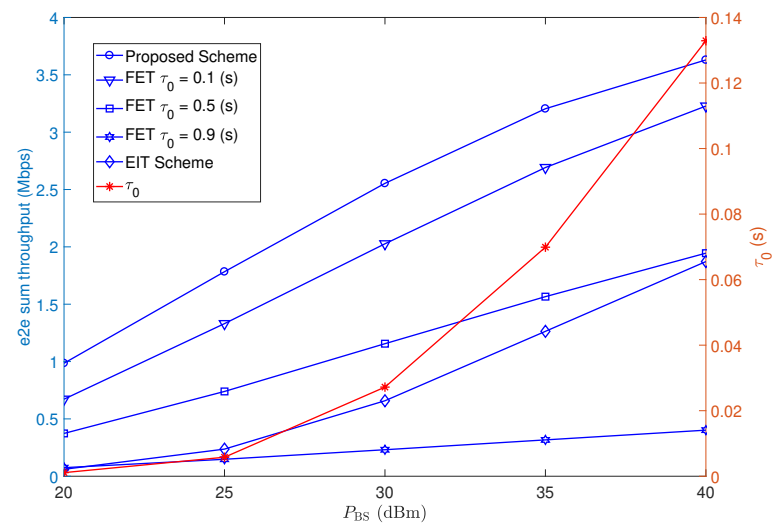

Fig. 3. The e2e sum throughput of the system for different schemes versus the transmit power of the BS, $K=10, N_{T}=4, P_{E}=30 \mathrm{dBm}$.

the EH duration of the FET is close to the optimal $\tau_{0}^{*}$ of the proposed scheme.

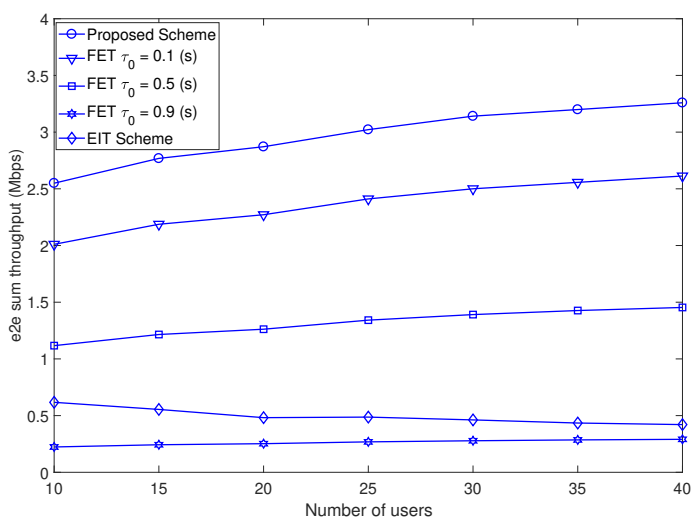

Fig. 4. The e2e sum throughput versus the number of users, with $P_{\mathrm{BS}}=$ $P_{E}=30 \mathrm{dBm}$, and $N_{T}=4$.

In Fig. 4, the e2e sum throughput of the schemes is evaluated with different numbers of users, where $P_{\mathrm{BS}}=P_{E}=30$ $\mathrm{dBm}$, and $N_{T}=4$. As can be seen that the e2e sum throughput for the FET and the proposed scheme is slightly increased, as the number of users increases. This is because a larger 
number of users leads to a higher multi-user diversity gain, which in turn results in a higher throughput of the system. However, there is a reverse trend in the performance of the EIT. This is because the duration of the WIT phase is divided into equal intervals, and consequently, resulting in insufficient time for the AP to serve all the users. Again, the proposed scheme achieves the best performance among all the considered schemes.

\section{CONCLUSION}

We investigated the problem of maximizing the e2e sum throughput of the AF-assisted RWPCN by jointly optimizing the power and time fraction for energy and information transmission. To address the nonconvex optimization problem, we first transformed this problem into a convex problem and then proposed an efficient iterative algorithm in which closedform solutions for the optimization variables were derived in each iteration. The desirability of jointly optimizing the ITA and EA was justified via numerical results. They also revealed that the proposed scheme obtains a fast convergence speed and outperforms the baseline schemes in terms of e $2 \mathrm{e}$ sum throughput. Interesting future works include: $i$ ) Optimal energy-efficient resource allocation for AF-assisted RWPCN networks, and ii) Advanced beamforming techniques at the AP and PS to further improve the system performance.

\section{APPENDIX A}

\section{PROOF OF PROPOSITION 1}

First, we show that each function in (T-P2) is jointly concave with respect to $e_{k}$ and $\tau_{k}$. Now, we prove the concavity of $R_{k}^{T}$ in (3) with the two variables $\tau_{k}$ and $e_{k}$ by checking the sign of its Hessian matrix $\mathbf{H}_{k}=\left[a_{k}, q_{k} ; q_{k}, b_{k}\right]$, where $a_{k}=-\Omega e_{k}^{2}$, $b_{k}=-\Omega \tau_{k}^{2}$ are the second-order partial derivatives of $R_{k}^{T}$ on $e_{k}, \tau_{k}$, respectively, and $q_{k}=\Omega \tau_{k} e_{k}$ is the second-order partial derivative of $R_{k}^{T}$ of each variable on the remaining variable and $\Omega=\frac{A C \gamma_{k}\left[2(A+B) B e_{k} \gamma_{k}^{2}+2 B C \gamma_{k} \tau_{k}+A C \tau_{k} \gamma_{k}\right]}{2\left[\left(B e_{k} \gamma_{k}+\tau_{k} C\right)\left((B+A) e_{k} \gamma_{k}+\tau_{k} C\right)\right]^{2}}$. It can be observed that $a_{k}=b_{k}\left(\frac{e_{k}}{\tau_{k}}\right)^{2}, q_{k}=-b_{k}\left(\frac{e_{k}}{\tau_{k}}\right)$. Note that there exists a real vector $t=\left[t_{1}, t_{2}\right]^{T}$ for

$$
\mathbf{t}^{T} \mathbf{H}_{k} \mathbf{t}=t_{1}^{2} a_{k}+2 t_{1} t_{2} q_{k}+t_{2}^{2} b_{k} .
$$

Then, $a_{k}$, and $b_{k}$ are substituted into (14), leading to $\mathbf{u}^{T} \mathbf{H}_{i} \mathbf{u}=$ $b_{k}\left(t_{1}\left(\frac{e_{k}}{\tau_{k}}\right)-t_{2}\right)^{2} \prec 0$, due to $b_{k} \leq 0$. Thus, $R_{k}^{T}$ is a concave function. The objective of (T-P2) has the form of summation of concave functions. It is also a joint function of concavity with $\tau_{k}$ and $e_{k}$. The proof of proposition 1 is completed.

\section{APPENDIX B}

\section{PROOF OF LEMMA 1}

The Lagrangian of (T-P3) is expressed as follows:

$$
\begin{aligned}
L_{e}(\mathbf{e}, \vartheta)= & W\left(\frac{1-\tau_{0}}{2}\right) \log \left(1+\frac{A \sum_{k=1}^{K} e_{k} \gamma_{k}}{B \sum_{k=1}^{K} e_{k} \gamma_{k}+\left(1-\tau_{0}\right) C}\right) \\
& +\vartheta\left(\Theta_{0} \tau_{0}-\sum_{k=1}^{K} e_{k}\right)
\end{aligned}
$$

where $\vartheta$ is a non-negative Lagrangian multiplier related to constraint $\overline{\mathrm{C}} 2$. We set the derivative of the Lagrangian to be zero to find the optimal $e_{k}$. After some mathematical manipulations, we obtain

$B(B+A) e_{k}^{2} \gamma_{k}^{2}+\left(\Phi_{k}(B+A)+B \Gamma_{k}\right) e_{k} \gamma_{k}+\Phi_{k} \Gamma_{k}-\Delta_{k}=0$,

where $\Delta_{k}=\frac{W A \gamma_{k}\left(1-\tau_{0}\right)^{2} C}{2 \vartheta}, \Phi_{k}=B \sum_{i=1, i \neq k}^{K} e_{i} \gamma_{i}+$ $\left(1-\tau_{0}\right) C$, and $\Gamma_{k}=\Phi_{k}+A \sum_{i=1, i \neq k}^{K} e_{i} \gamma_{i}$. Let denote $Q_{k}=\left(\Phi_{k}(B+A)+B \Gamma_{k}\right) \gamma_{k}, U_{k}=\left(\Phi_{k} \Gamma_{k}-\Delta_{k}\right)$, and $\Psi_{k}=B(B+A) \gamma_{k}^{2}$. By solving (16), we obtain the optimal $\mathrm{EA}$ as in (12) and (13).

\section{REFERENCES}

[1] I. Krikidis, S. Timotheou, S. Nikolaou, G. Zheng, D. W. K. Ng, and R. Schober, "Simultaneous wireless information and power transfer in modern communication systems," IEEE Communications Magazine, vol. 52, no. 11, pp. 104-110, Nov. 2014.

[2] V. Nguyen, T. Q. Duong, H. D. Tuan, O. Shin, and H. V. Poor, "Spectral and energy efficiencies in full-duplex wireless information and power transfer," IEEE Transactions on Communications, vol. 65, no. 5, pp. 2220-2233, 2017.

[3] T. Nguyen, V. Nguyen, J. Lee, and Y. Kim, "Sum rate maximization for multi-user wireless powered IoT network with non-linear energy harvester: Time and power allocation," IEEE Access, vol. 7, pp. $149698-$ 149710, 2019.

[4] X. Zhou, J. Guo, S. Durrani, and M. D. Renzo, "Power beaconassisted millimeter wave ad hoc networks," IEEE Transactions on Communications, vol. 66, no. 2, pp. 830-844, Feb. 2018.

[5] J. Guo, X. Zhou, and S. Durrani, "Wireless power transfer via mmWave power beacons with directional beamforming," IEEE Wireless Communications Letters, vol. 8, no. 1, pp. 17-20, Feb. 2019.

[6] P. Ramezani, Y. Zeng, and A. Jamalipour, "Optimal resource allocation for multiuser Internet of things network with single wireless-powered relay," IEEE Internet of Things Journal, vol. 6, no. 2, pp. 3132-3142, Apr. 2019.

[7] G. Hu and Y. Cai, "Energy-accumulation-based power-beacon-assisted WPCN: Time allocation and scheduling schemes," IEEE Communications Letters, vol. 23, no. 9, pp. 1510-1514, Sep. 2019.

[8] H. Yu, M. Ju, and C. Jeong, "Sum-throughput maximization in dual-hop wireless powered DF-based relay networks," IEEE Access, vol. 7, pp. $162268-162281,2019$

[9] V.-P. Bui, V.-D. Nguyen, H. V. Nguyen, O. A. Dobre, and O.-S. Shin, "Optimization of rate fairness in multi-pair wireless-powered relaying systems," IEEE Communications Letters, vol. 24, no. 3, pp. 603-607, Mar. 2020.

[10] E. Boshkovska, D. W. K. Ng, N. Zlatanov, and R. Schober, "Practical non-linear energy harvesting model and resource allocation for SWIPT systems," IEEE Communications Letters, vol. 19, no. 12, pp. 2082-2085, Dec. 2015.

[11] E. Boshkovska, R. Morsi, D. W. K. Ng, and R. Schober, "Power allocation and scheduling for SWIPT systems with non-linear energy harvesting model," in 2016 IEEE International Conference on Cоттиnications (ICC), Kuala Lumpur, Malaysia, 2016, pp. 1-6.

[12] J. Tang, J. Luo, M. Liu, D. K. C. So, E. Alsusa, G. Chen, K. Wong, and J. A. Chambers, "Energy efficiency optimization for NOMA with SWIPT," IEEE Journal of Selected Topics in Signal Processing, vol. 13, no. 3, pp. 452-466, Jun. 2019. 